\title{
Mortality and Causes of Death
}

\section{among Burn Patients at the Burn Unit of Cipto Mangunkusumo Hospital}

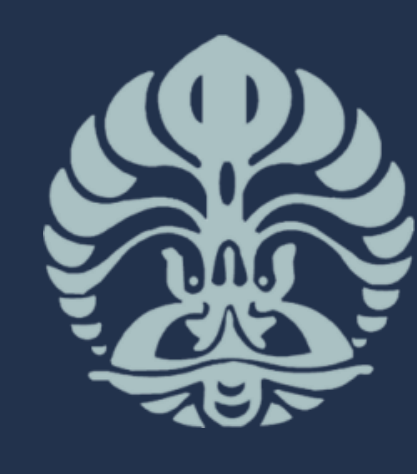

\section{Aditya Wardhana, Radhianie Djan, Zainul Halim}

Introduction

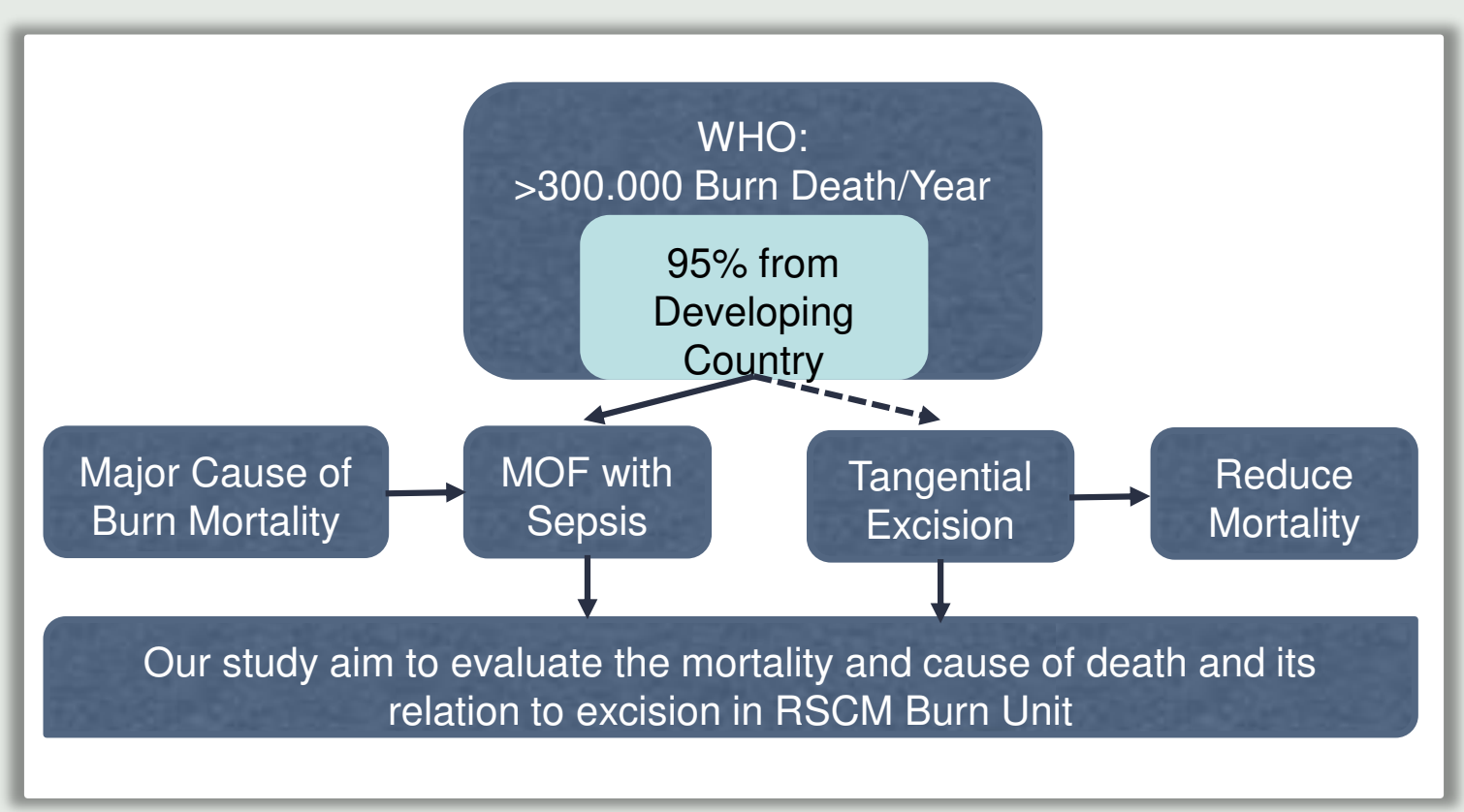

Method

A retrospective analysis from the medical records of Cipto Mangunkusumo hospital burn unit between January 2013 - June 2017 $(n=157)$.

We collect the demographic data of mortality, causes of death, and the patterns of bacterial isolates among deceased patients (only sepsis) in our unit.

Statistical analysis was performed using SPSS ver. 23.0. MannWhitney and Unpaired T test was used to analyze non parametric data. Fischer's Exact and Chi Square test was used for qualitative data. The data was considered statistically significant if $p$-value less than 0,05 .

\section{Results}

Figure 1. Total of Patients Admitted and Died in RSCM Burn Unit 2013-2017

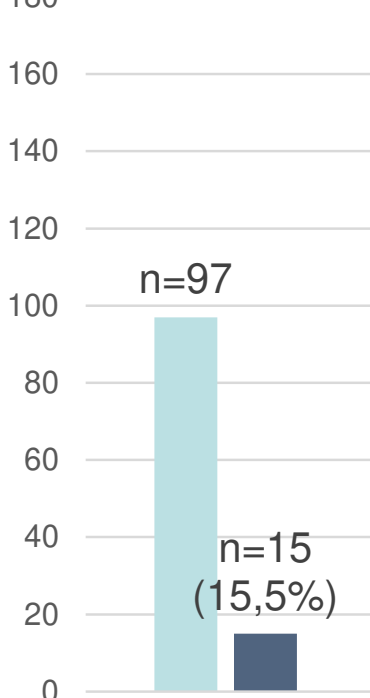

2013

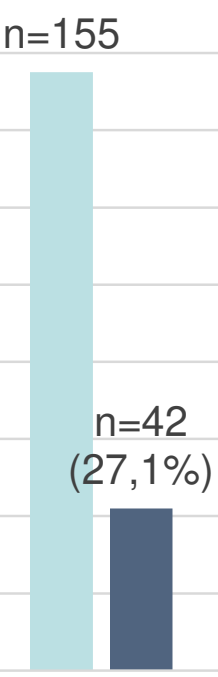

2014

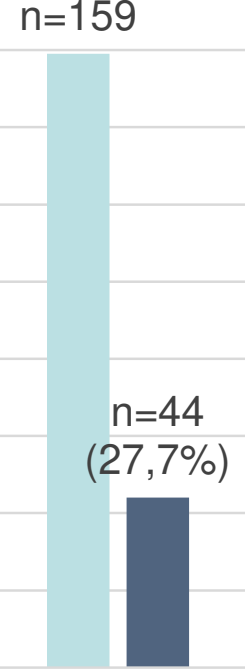

2015 $n=146$

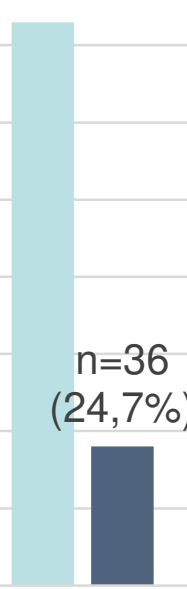

2016

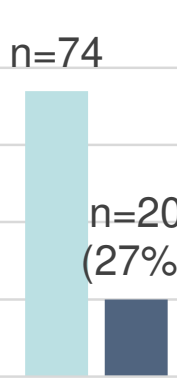

2017

Patients Admitted $\quad$ Patient Died

Table 1. Characteristics of Deceased Burn Patients in RSCM Burn Unit

\begin{tabular}{|c|c|c|c|}
\hline \multirow[b]{2}{*}{ Characteristics } & \multicolumn{2}{|c|}{ Tangential Excision } & \multirow[b]{2}{*}{ p-Value } \\
\hline & $\begin{array}{c}\text { Performed } \\
\quad(n=89)\end{array}$ & $\begin{array}{c}\text { Not } \\
\text { Performed } \\
(n=68)\end{array}$ & \\
\hline Age (years), mean (SD) & $34,6(17,1)$ & $\begin{array}{l}39,39 \\
(18,6)\end{array}$ & 0,097 \\
\hline $\begin{array}{l}\text { Gender, n (\%) } \\
\text { Male } \\
\text { Female }\end{array}$ & $\begin{array}{l}60(67,4) \\
29(32,6)\end{array}$ & $\begin{array}{l}42(61,8) \\
26(38,2)\end{array}$ & 0,502 \\
\hline $\begin{array}{l}\text { Inhalation Injury, n (\%) } \\
\text { Yes } \\
\text { No }\end{array}$ & $\begin{array}{l}37(41,6) \\
52(58,4)\end{array}$ & $\begin{array}{l}30(44,1) \\
38(55,9)\end{array}$ & 0,871 \\
\hline $\begin{array}{l}\text { Length of Stay (days), median } \\
\text { (range) }\end{array}$ & $11(1-81)$ & $6,5(1-24)$ & $<0.001^{*}$ \\
\hline TBSA, mean (SD) & $54,1(20,4)$ & $59,4(24,0)$ & 0,133 \\
\hline $\begin{array}{l}\text { Mechanism of Injury } \mathbf{n}(\%) \\
\text { Flame } \\
\text { Blast/ Gas Injury } \\
\text { Others } \\
\text { Electrical } \\
\text { Chemical } \\
\text { Scald }\end{array}$ & $\begin{array}{c}70(78,7) \\
45(64,3) \\
25(35,7) \\
11(12,4) \\
3(3,4) \\
5(5,6)\end{array}$ & $\begin{array}{c}57(83,8) \\
26(45,6) \\
31(54,4) \\
4(5,9) \\
2(2,9) \\
5(7,4)\end{array}$ & $\begin{array}{l}0,273 \\
1,000 \\
0,502\end{array}$ \\
\hline
\end{tabular}

Table 2. Causes of Death in RSCM Burn Unit

\begin{tabular}{lccc}
\hline \multirow{1}{*}{ Cause of Death } & \multicolumn{2}{c}{ Tangential Excision } & \\
\cline { 2 - 3 } & $\begin{array}{c}\text { Performed } \\
(\mathrm{n}=89) \\
\mathrm{n}(\%)\end{array}$ & $\begin{array}{c}\text { Not Performed } \\
(\mathrm{n}=68) \\
\mathrm{n}(\%)\end{array}$ & p-Value \\
\hline $\begin{array}{l}\text { Sepsis } \\
\begin{array}{l}\text { Multiple Organ } \\
\text { Failure (MOF) }\end{array}\end{array}$ & $31(34,8)$ & $32(47,1)$ & 0,141 \\
\hline $\begin{array}{l}\text { Acute Respiratory } \\
\text { Distress Syndrome } \\
\text { (ARDS) }\end{array}$ & $15(16,9)$ & $21(30,9)$ & 0,139 \\
$\begin{array}{l}\text { Acute Kidney Injury } \\
\text { (AKI) }\end{array}$ & $3(3,4)$ & $13(19,1)$ & 0,834 \\
\hline Hypovolemic Shock & $2(2,2)$ & $1(1,5)$ & 1,000 \\
\hline
\end{tabular}

Table 3. Patterns of Bacterial Isolates among Deceased Sepsis Patients in RSCM Burn Unit

\begin{tabular}{lccc}
\hline \multirow{1}{*}{ Organisms } & \multicolumn{2}{c}{ Tangential Excision } & \\
\cline { 2 - 3 } & $\begin{array}{c}\text { Performed } \\
(\mathrm{n}=26) \\
\mathrm{n}(\%)\end{array}$ & $\begin{array}{c}\text { Not Performed } \\
(\mathrm{n}=26) \\
\mathrm{n}(\%)\end{array}$ & p-Value \\
\hline Klebsiella Pneumoniae & $13(50,0)$ & $13(50,0)$ & 1,000 \\
Pseudomonas Aeruginosa & $15(57,7)$ & $9(34,6)$ & 0,164 \\
Acinetobacter Baumannii & $10(38,5)$ & $10(38,5)$ & 1,000 \\
Enterobacter Aerogenes & $5(19,2)$ & $8(30,8)$ & 0,523 \\
Escherichia Coli & $0(0)$ & $5(20)$ & $0,023^{*}$ \\
Staphylococcus Epidermidis & $0(0)$ & $3(11,5)$ & 0,235 \\
Candida sp. & $3(11,5)$ & $2(7,7)$ & 1,000 \\
Streptococcus sp. & $0(0)$ & $4(15,4)$ & 0,110 \\
Proteus Mirabilis & $1(3,8)$ & $1(3,8)$ & 1,000 \\
Enterococcus Faecalis & $0(0)$ & $1(3,8)$ & 1,000 \\
Enterobacter cloacae & $2(7,7)$ & $1(3,8)$ & 1,000 \\
\hline Staphylococcus Saprophyticus & $1(3,8)$ & $1(3,8)$ & 1,000 \\
\hline
\end{tabular}

\section{Conclusion}

Primary features of burn deceased patients in our setting are commonly found among males within working age with the major cause of burn are flames particularly due to blast injury and sepsis was found as the major cause of death. These findings are concurrent with the available literature related to mortality in burns.

However, despite its popular belief, our data suggests tangential excision procedure does not significantly affect the pattern of mortality and cause of death among burn patients in our setting. Length of hospitalization is shown to be longer among eighty-nine patients who underwent excision before death. Positive bacterial culture is also shown to have no significant differences between excision and non-excision group diagnosed with sepsis.

One of the factors that may contribute to these findings are the timing of excision which mostly delay due to late admission in our burn centre. Furthermore, most admitted patients in our setting have greater than $50 \%$ TBSA and almost $50 \%$ of deceased patients have inhalation injury.

\section{References}

World Health Organization. A WHO plan for burn prevention and care 20089 February 2017. Available from:

http://whqlibdoc.who.int/publications/2008/97892415 96299 eng.pdf

Forjuoh SN. Burns in low- and middle-income countries: a review of available literature on descriptive epidemiology, risk factors, treatment, and prevention. Burns. 2006;32(5):529-37.

Bloemsma GC, Dokter J, Boxma H, Oen IM Mortality and causes of death in a burn centre. Burns. 2008;34(8):1103-7.

4. Kallinen O, Maisniemi K, Bohling T, Tukiainen E, Koljonen V. Multiple organ failure as a cause of death in patients with severe burns. J Burn Care Res. 2012;33(2):206-11. 